\title{
Metabolic engineering of Escherichia coli for production of mixed isoprenoid alcohols and their derivatives
}

\author{
Bakht Zada ${ }^{1 \dagger}$, Chonglong Wang ${ }^{2 \dagger}$, Ji-Bin Park', Seong-Hee Jeong ${ }^{1}$, Ju-Eon Park', Hawaibam Birla Singh ${ }^{1}$ \\ and Seon-Won Kim ${ }^{1 *}$
}

\begin{abstract}
Background: Current petroleum-derived fuels such as gasoline $\left(C_{5}-C_{12}\right)$ and diesel $\left(C_{15}-C_{22}\right)$ are complex mixtures of hydrocarbons with different chain lengths and chemical structures. Isoprenoids are hydrocarbon-based compounds with different carbon chain lengths and diverse chemical structures, similar to petroleum. Thus, isoprenoid alcohols such as isopentenol $\left(C_{5}\right)$, geraniol $\left(C_{10}\right)$, and farnesol $\left(C_{15}\right)$ have been considered to be ideal biofuel candidates. NudB, a native phosphatase of Escherichia coli, is reported to dephosphorylate isopentenyl diphosphate (IPP) and dimethylallyl diphosphate (DMAPP) into isopentenol. However, no attention has been paid to its promiscuous activity toward longer chain length $\left(\mathrm{C}_{10}-\mathrm{C}_{15}\right)$ prenyl diphosphates.

Results: In this study, the promiscuous activity of NudB toward geranyl diphosphate (GPP) and farnesyl diphosphate (FPP) was applied for the production of isoprenoid alcohol mixtures, including isopentenol, geraniol, and farnesol, and their derivatives. E. coli was engineered to produce a mixture of $C_{5}$ and $C_{15}$ alcohols by overexpressing NudB (dihydroneopterin triphosphate diphosphohydrolase) and IspA (FPP synthase) along with a heterologous MVA pathway, which resulted in a total of up to $1652 \mathrm{mg} / \mathrm{L}$ mixture of $C_{5}$ and $C_{15}$ alcohols and their derivatives. The production was further increased to $2027 \mathrm{mg} / \mathrm{L}$ by overexpression of another endogenous phosphatase, AphA, in addition to NudB. Production of DMAPP- and FPP-derived alcohols and their derivatives was significantly increased with an increase in the gene dosage of idi, encoding IPP isomerase (IDI), indicating a potential modulation of the composition of the alcohols mixture according to the expression level of IDI. When IspA was replaced with its mutant IspA*, generating GPP in the production strain, a total of $1418 \mathrm{mg} / \mathrm{L}$ of the isoprenoid mixture was obtained containing $\mathrm{C}_{10}$ alcohols as a main component.
\end{abstract}

Conclusions: The promiscuous activity of NudB was newly identified and successfully used for production of isoprenoid-based alcohol mixtures, which are suitable as next-generation biofuels or commodity chemicals. This is the first successful report on high-titer production of an isoprenoid alcohol-based mixture. The engineering approaches can provide a valuable platform for production of other isoprenoid mixtures via a proportional modulation of IPP, DMAPP, GPP, and FPP syntheses.

Keywords: Isoprenoid mixture, Isopentenol, Geraniol, Farnesol, Escherichia coli, NudB

\footnotetext{
*Correspondence: swkim@gnu.ac.kr

†Bakht Zada and Chonglong Wang contributed equally to this work

${ }^{1}$ Division of Applied Life Science (BK21 Plus Program), PMBBRC,

Gyeongsang National University, Jinju 52828, Republic of Korea

Full list of author information is available at the end of the article
} 


\section{Background}

Petroleum-derived fuels used for transportation are complex mixtures of carbon compounds with different chain lengths and chemical structures. For example, gasoline, diesel, and jet fuels are mixture of hydrocarbons with carbon number distribution from 5 to $12,15-22$, and $8-16$, respectively. With the increasing concern about the limited supply of petroleum and the effects of climate change [1], interest in researching microbial production of sustainable and renewable biofuels has increased [2]. Advanced biofuels are required to have similar fuel properties to petroleum-derived gasoline, diesel, or jet fuels in order to replace the current fuels [3-5].

Isoprenoids, also known as terpenoids, are a large and diverse class of natural compounds used as pharmaceuticals, fragrances and flavors, solvents, and more recently, potential advanced biofuels [6-9]. Isopentenol $\left(\mathrm{C}_{5} \mathrm{H}_{10} \mathrm{O}\right.$, also known as isoprenol and prenol), geraniol $\left(\mathrm{C}_{10} \mathrm{H}_{18} \mathrm{O}\right)$, and farnesol $\left(\mathrm{C}_{15} \mathrm{H}_{26} \mathrm{O}\right)$ are important acyclic hemiterpene, monoterpene, and sesquiterpene alcohols, respectively. These alcohols are considered to be advanced biofuels due to their high-energy content, low hygroscopicity, and low volatility [2, 10-13]. Additionally, they have a wide range of commercial applications as flavor, fragrance, pharmaceuticals, biopesticides, and precursors to important industrial chemicals [7, 14-23]. Isopentenol, geraniol, and farnesol are biologically generated from prenyl diphosphates precursors, IPP/DMAPP, GPP, and FPP, respectively. IPP and DMAPP are synthesized via the methylerythritol phosphate (MEP) pathway, starting from condensation of pyruvate and glyceraldehyde-3-phosphate (G-3-P) in most prokaryotes including E. coli, and the mevalonate (MVA) pathway, commencing with condensation of three acetyl-CoA in eukaryotes [24, 25]. GPP and FPP are synthesized by an isoprenyl diphosphate synthase such as IspA via a sequential head-to-tail condensation of IPP and DMAPP.

The dephosphorylation of IPP/DMAPP, GPP, and FPP to isopentenol, geraniol, and farnesol, respectively can be catalyzed by various phosphatases, diphosphatases, and terpene synthases. Some of $E$. coli endogenous phosphatases efficiently catalyze the dephosphorylation of IPP and DMAPP to isopentenol [13], but an effective native enzyme, catalyzing GPP and FPP to geraniol and farnesol, respectively, has not yet been identified. Exogenous geraniol synthase from Ocimum basilicum has been expressed for geraniol production in E. coli [26-28]. Bifunctional diacylglycerol diphosphate phosphatase (DPP1) and lipid phosphate phosphatase (LPP1) from Saccharomyces cerevisiae have been evaluated for geraniol biosynthesis [29]. The endogenous E. coli ADP-ribose pyrophosphatase (NudF) and alkaline phosphatase (PhoA) were also tested for geraniol production; however, only a tiny amount $(5.3 \mathrm{mg} / \mathrm{L})$ of geraniol was produced by PhoA in a flask culture [29]. Farnesyl phosphatases from Homo sapiens and Drosophila melanogaster can hydrolyze FPP to farnesol [30, 31]. Farnesol synthase from Oryza sativa and sesquiterpene synthase from Zea mays catalyze the conversion of FPP to farnesol $[32,33]$. Several phosphatases from S. cerevisiae such as a truncated alkaline phosphatase, diacylglycerol diphosphate phosphatase, and lipid phosphate phosphatase show specificity toward FPP [34, 35]. E. coli native phosphatases, PhoA, AphA, BacA, PgpA, and PgpB, have also been assessed for farnesol synthesis [36, 37]. However, the enzymes investigated for farnesol synthesis have low substrate specificity, low catalytic activity, or poor protein expression in microbial hosts. Thus, the discovery of a promising candidate enzyme that can effectively catalyze dephosphorylations of prenyl diphosphates to their corresponding alcohols is still needed.

Nudix hydrolases, which are distributed among all kingdoms of life, potentially recognize and dephosphorylate phosphate moieties of a wide range of phosphorylated compounds [38, 39]. Nudix hydrolases from Bacillus subtilis (BsNudF) and E. coli (NudB, NudF, NudI, NudJ, and NudM) convert IPP and DMAPP into isopentenol [13, 40]. As Nudix hydrolases have been suspected to dephosphorylate isopentenyl diphosphates to isoprenoid alcohols, the highly active NudB is used here for production of a mixture of isoprenoid alcohols including geraniol, farnesol, and isopentenol (Fig. 1a).

\section{Results and discussion}

Engineering a synthetic pathway for a mixture of isoprenoid-based $C_{5}$ and $C_{15}$ alcohols

In order to convert IPP/DMAPP to isopentenol and FPP to farnesol in E. coli, it is necessary to overexpress endogenous or exogenous phosphatases for dephosphorylation of these prenyl diphosphates. The endogenous phosphatase NudB effectively hydrolyzes IPP and DMAPP to isopentenol [13, 41-43]. As Nudix hydrolases generally show broad substrate specificities [38], we hypothesized that NudB could also have catalytic activity toward FPP. Thus, $n u d B$ was cloned with $i s p A$ encoding FPP synthase into a strong expression vector, $\mathrm{p} \operatorname{Trc} 99 \mathrm{~A}$, resulting in plasmid pT-MBF1.0 (Fig. 1b). Plasmid pSNA, expressing an exogenous mevalonate (MVA) pathway, was used to effectively supply prenyl diphosphate precursors for synthesis of mixture isoprenoid alcohols (Fig. 1b) [44]. Both of the plasmids were transformed into E. coli DH5 $\alpha$, resulting in strain NA-MBF1.0 (Additional file 1: Table S1). After culture of the strain for $48 \mathrm{~h}$, the culture broth was analyzed by gas chromatography (GC), which showed seven peaks that were subsequently identified through gas chromatography-mass spectrometry 


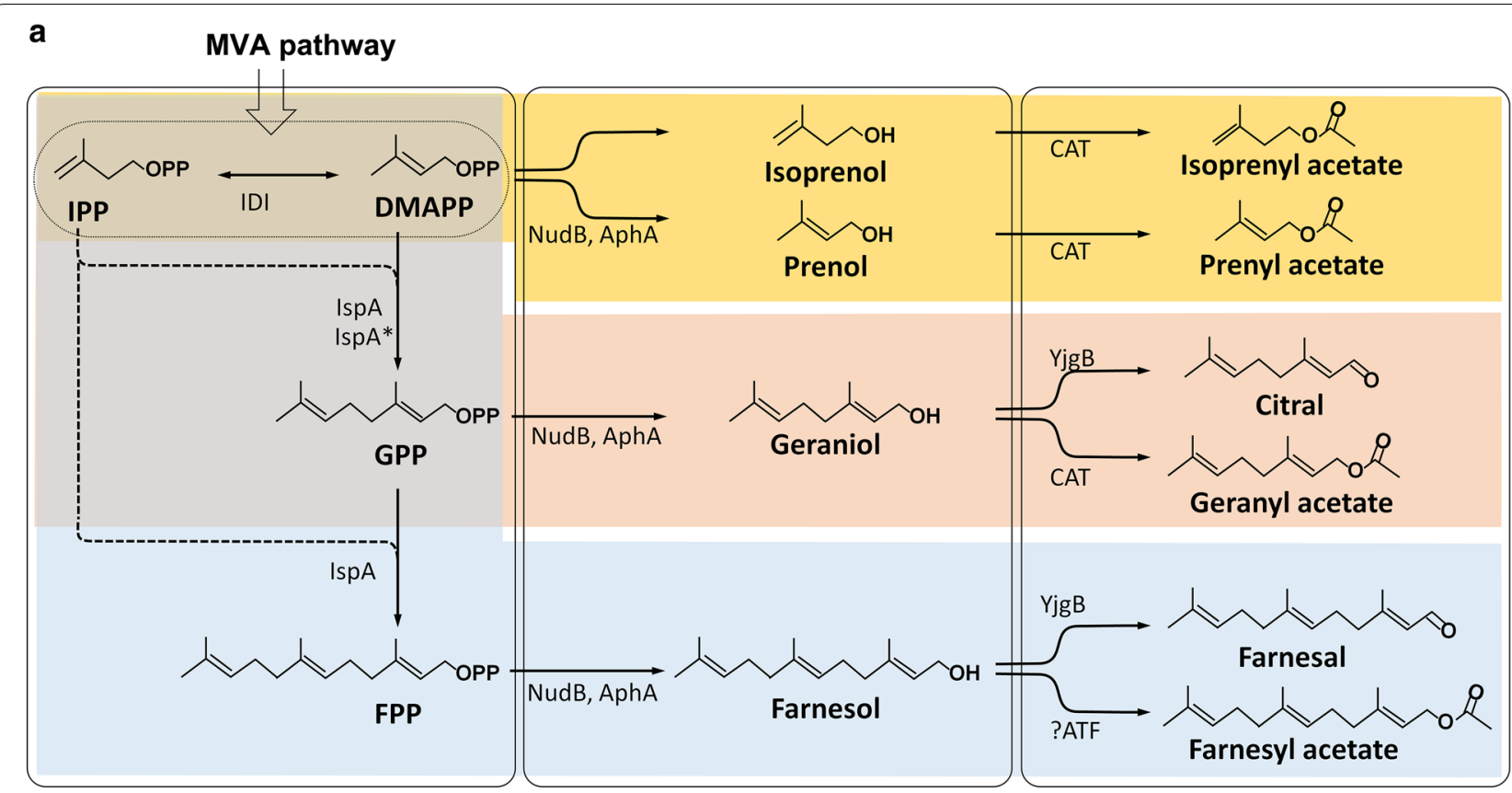

b
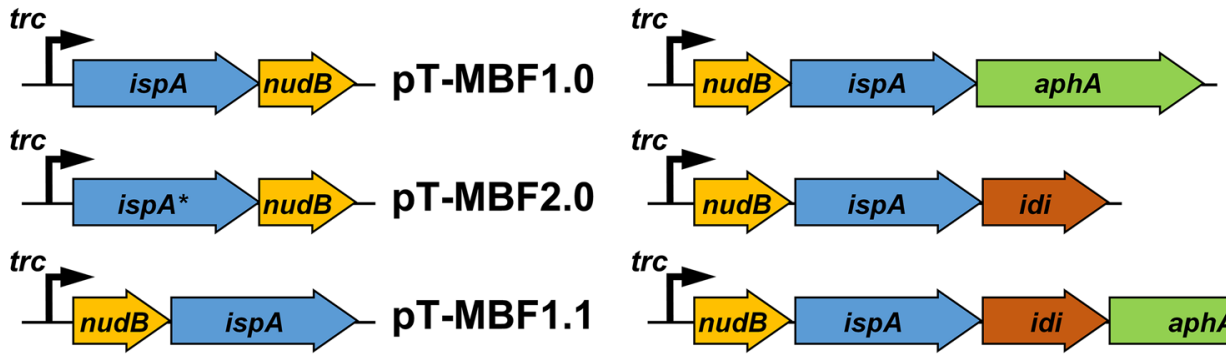

pT-MBF1.2
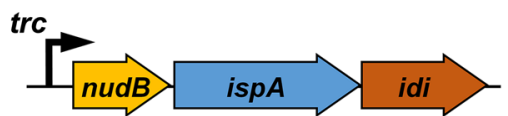

pT-MBF1.1a

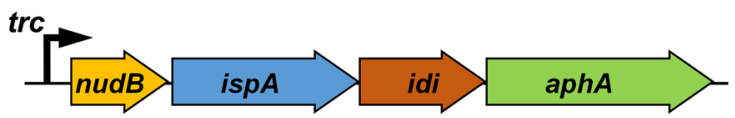

pT-MBF1.2a
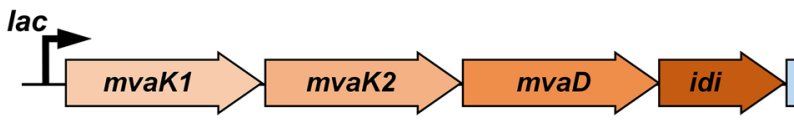

mvaE

mvas

pSNA / pSNAK

Fig. 1 Schematic overview of synthesis pathway (a) and expression constructs containing the pathway genes (b). GPP and FPP are synthesized by IspA* and IspA, respectively, through condensations of DMAPP and IPP, generated from the MVA pathway. NudB catalyzes dephosphorylation of IPP, DMAPP, GPP, and FPP into IP, DMAP, GP, and FP, respectively. AphA catalyzes hydrolysis of IP, DMAP, GP, and FP into isoprenol, prenol, geraniol, and farnesol, respectively. Chloramphenicol acetyltransferase (CAT) is responsible for transformation of isoprenol, prenol, and geraniol into their corresponding acetate esters. An alcohol dehydrogenase, YjgB oxidizes geraniol and farnesol into citral and farnesal, respectively. An unknown acetyl transferase (ATF) is involved in transformation of farnesol into farnesyl acetate. Solid colored arrows and black bent arrows represent genes and promoters, respectively. Abbreviations of the pathway intermediates are as follows: MVA, mevalonate; IPP, isopentenyl diphosphate; DMAPP, dimethylallyl diphosphate; GPP, geranyl diphosphate; FPP, farnesyl diphosphate; IP, isopentenyl phosphate; DMAP, dimethylallyl phosphate; GP, geranyl phosphate; FP, farnesyl phosphate. The abbreviations of enzymes are as follows: NudB, dihydroneopterin triphosphate diphosphatase; IspA, farnesyl diphosphate synthase; AphA, acid phosphatase; IspA*, IspA mutant of geranyl diphosphate synthase; MvaE, bifunctional acetoacetyl-CoA thiolase and HMG-CoA reductase; MvaS, HMG-CoA synthase; MvaK1, mevalonate kinase; MvaK2, phosphomevalonate kinase; MvaD, mevalonate diphosphate decarboxylase; IDI, isopentenyl diphosphate isomerase; CAT, chloramphenicol acetyltransferase; YjgB NADPH-dependent aldehyde reductases; ?ATF, unknown acetyl transferase of E. coli

(GC-MS) (Fig. 2; Additional file 1: Fig. S1). Three of the seven peaks were identified to be isoprenol, prenol $\left(\mathrm{C}_{5}\right.$ alcohols), and farnesol ( $\mathrm{C}_{15}$ alcohol) as expected (Fig. 2).
The other four unexpected peaks corresponded to isoprenyl acetate, farnesyl acetate, $Z, E$-farnesal, and $E, E$ farnesal (Fig. 2). Thus, the strain NA-MBF1.0 successfully 

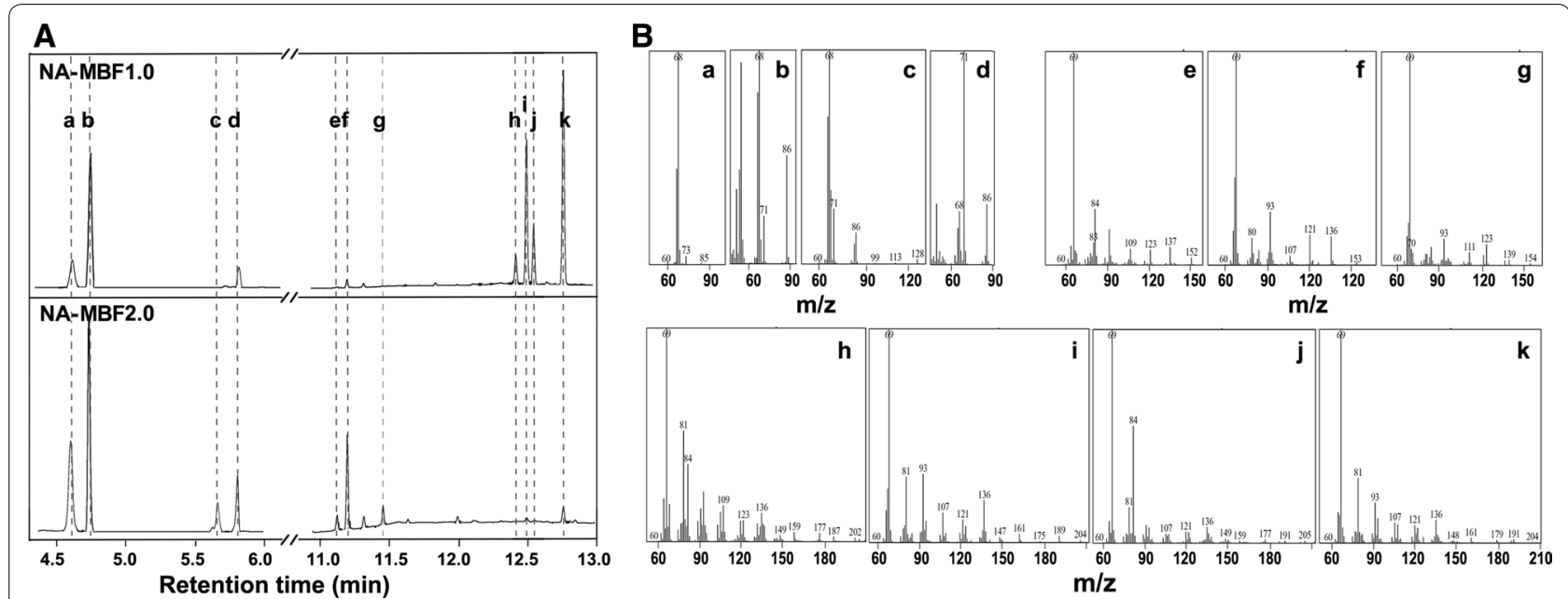

Fig. 2 GC-FID and GC-MS analysis of $C_{5}, C_{10}$, and $C_{15}$ isoprenoid alcohols and their derivatives from engineered E. coli strains. GC-FID chromatograms (A) and mass spectra (B) of culture extracts obtained from recombinant E. coli strains NA-MBF1.0 and NA-MBF2.0. The GC-FID peaks and their corresponding compound are a isoprenyl acetate; $b$ isoprenol; c prenyl acetate; $d$ prenol; e citral; $f$ geranyl acetate; $g$ geraniol; $h$ Z,E-farnesal; i farnesyl acetate; $\mathrm{j}$,E-farnesal; and $\mathrm{k}$ farnesol produced $221 \mathrm{mg} / \mathrm{L}$ of $\mathrm{C}_{5}$ alcohols and their derivatives, and $626 \mathrm{mg} / \mathrm{L}$ of a $\mathrm{C}_{15}$ alcohol and its derivatives, which was a total of $847 \mathrm{mg} / \mathrm{L}$ isoprenoid biofuel mixture (Fig. 3a). In addition to $\mathrm{C}_{5}$ products, $65.1 \%$ of the total isoprenoid mixture produced in the strain NA-MBF1.0 was farnesol, a fourfold increase over the reference strain (135.5 mg/L) overexpressing IspA only [37]. Previously, the overexpression of $\mathrm{NudB}$ was used for the production of $\mathrm{C}_{5}$ products, isopentenol, because it was considered to have its activity only to IPP/DMAPP [13, 41-43]. However, our result indicates that NudB is able to dephosphorylate FPP to farnesol ( $\mathrm{C}_{15}$ products), as well as IPP/ DMAPP to isopentenol. NudB was thus presumed to be a potential phosphatase, hydrolyzing a wide range of prenyl diphosphates, and is likely appropriate for production of a mixture of isoprenoid alcohols as biofuel. The new promiscuous activity of NudB toward FPP was verified by in vitro study (Additional file 2: Fig. S6).

\section{Engineering a synthetic pathway for a mixture of isoprenoid-based $C_{5}$ and $C_{10}$ alcohols}

The recombinant $E$. coli strain overexpressing IspA and NudB successfully produced $C_{5}$ and $C_{15}$ isoprenoid alcohols, but not the $\mathrm{C}_{10}$ isoprenoid alcohol. This is probably due to absence of a GPP synthase (GPPS) to catalyze the condensation of DMAPP and IPP to GPP, the universal precursor of all monoterpenes $\left(\mathrm{C}_{10}\right)$ (Fig. 1a). Thus, production of $\mathrm{C}_{10}$ isoprenoid alcohol in E. coli has been limited due to poor supply of GPP. E. coli IspA was mutated to release GPP precursor for monoterpene biosynthesis [45]. The mutant IspA (IspA*), with a mutation of serine to phenylalanine at the 80th residue, catalyzes a single condensation of IPP and DMAPP, unlike wild-type IspA that catalyzes two sequential condensations of two IPPs with DMAPP. Overexpression of IspA* and the geraniol synthase of $O$. basilicum in recombinant $E$. coli harboring a heterologous MVA pathway was reported to produce $182 \mathrm{mg} / \mathrm{L}$ of geraniol [26]. Thus, IspA* was also used in this study to supply GPP to evaluate the activity of NudB for production of $\mathrm{C}_{10}$ isoprenoid alcohol. Plasmid pT-MBF2.0 was constructed by replacement of the ispA gene on plasmid pT-MBF1.0 with isp $A^{*}$ (Fig. 1b). E. coli strain NA-MBF2.0 harboring pT-MBF2.0 and pSNA produced geraniol and its derivatives (citral and geranyl acetate) as well as isoprenol, isoprenyl acetate, prenol, prenyl acetate, and farnesol (Fig. 2; Additional file 1: Fig. S1). A total concentration of $1418 \mathrm{mg} / \mathrm{L}$ of $\mathrm{C}_{5}$ and $\mathrm{C}_{10}$ products with a small amount $(12 \mathrm{mg} / \mathrm{L})$ of $\mathrm{C}_{15}$ product was produced from the strain NA-MBF2.0 after $48 \mathrm{~h}$ of culture (Fig. $3 \mathrm{~b}$; Table 1 ). The $\mathrm{C}_{5}$ family products were a major fraction of the isoprenoid mixture, but the $\mathrm{C}_{10}$ and $\mathrm{C}_{15}$ family products were minor components (Fig. $3 \mathrm{~b}$ ). The amount of $\mathrm{C}_{10}$ family products $(99 \mathrm{mg} / \mathrm{L})$ obtained by overexpression of IspA* in strain NA-MBF2.0 was significantly lower than that of the $\mathrm{C}_{15}$ family products $(626 \mathrm{mg} / \mathrm{L})$ from strain NA-MBF1.0 with overexpression of IspA. This is probably due to the lower catalytic activity of IspA* compared to wild-type IspA. Thus, the lower production of $\mathrm{C}_{10}$ family products in strain NAMBF2.0 could be attributed to a poor supply of GPP. An effective GPPS is necessary for sufficient supply of GPP to increase $\mathrm{C}_{10}$ products in the isoprenoid mixture. No study has previously focused on the promiscuous activity of NudB toward GPP. Our findings showed that NudB 

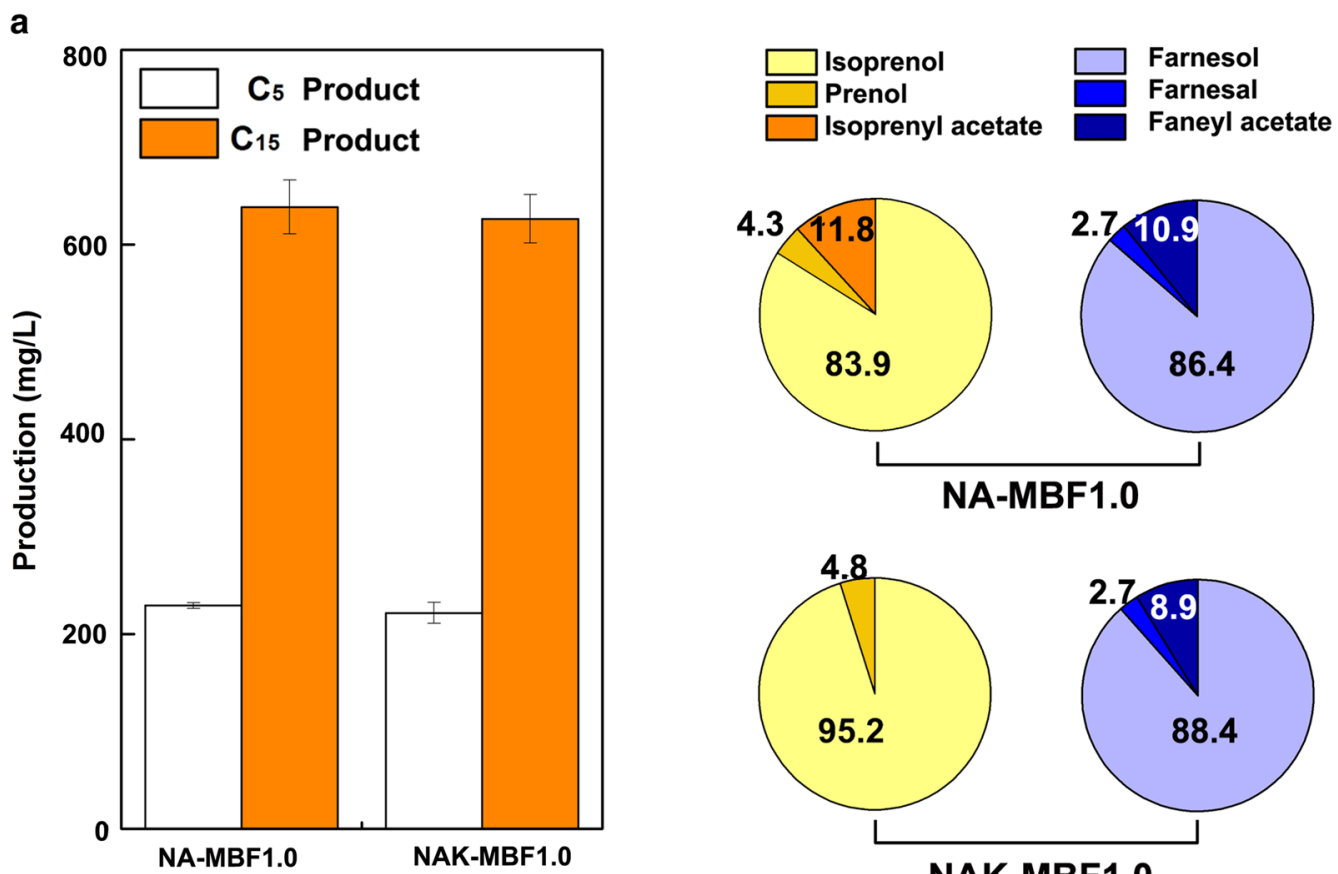

NA-MBF1.0
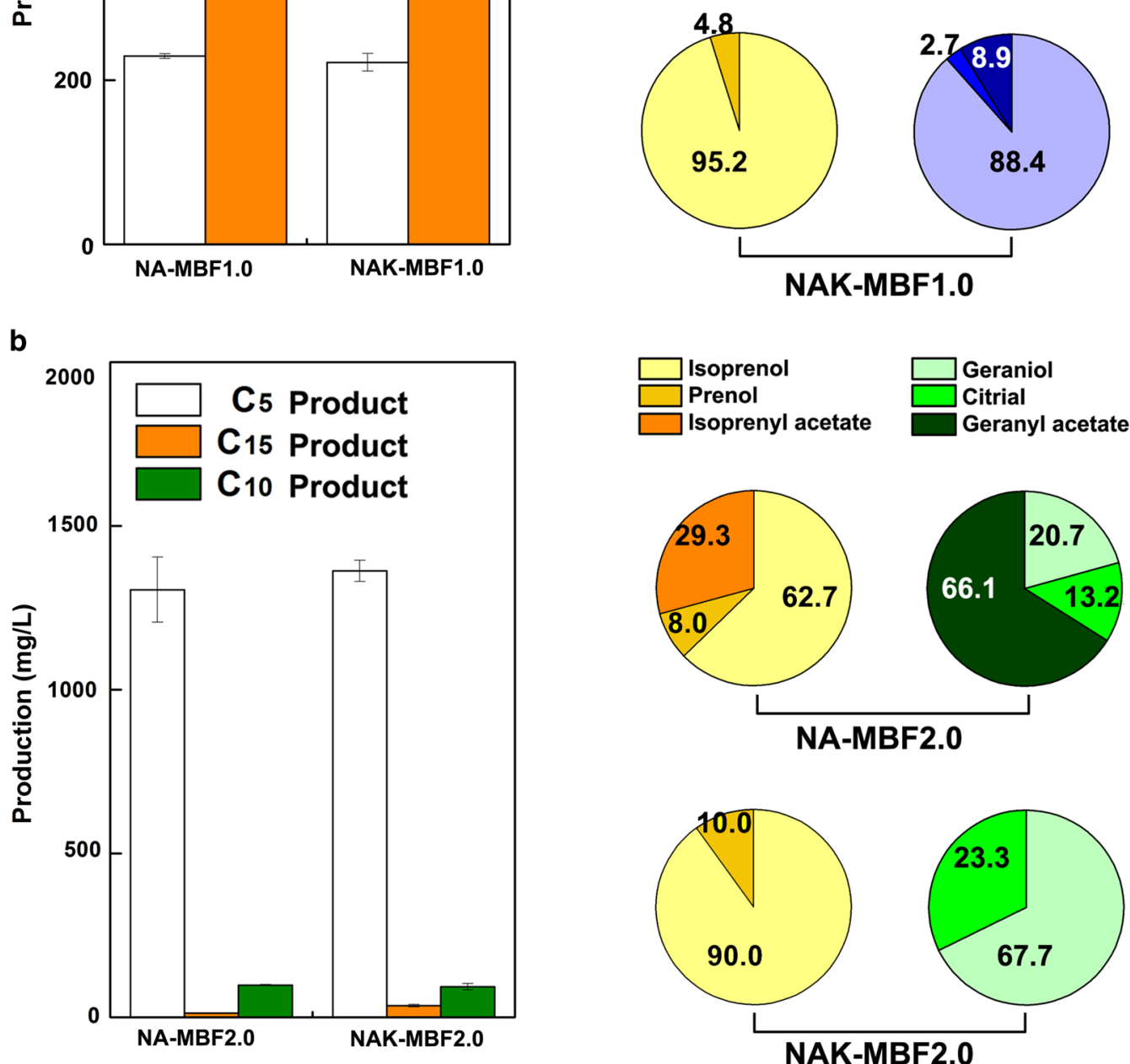

Fig. 3 Production of $C_{5}, C_{10}$, and $C_{15}$ isoprenoid alcohols and their derivatives in engineered E. coli. a Production and fractional compositions (\%) of IPP/DMAPP $\left(\mathrm{C}_{5}\right)$ - and FPP $\left(\mathrm{C}_{15}\right)$-derived isoprenoids from strains NA-MBF1.0 and NAK-MBF1.0. Strains NA-MBF1.0 and NAK-MBF1.0 are E. coli DH5a strains harboring pSNA and pSNAK, respectively, along with pT-MBF1.0. b Production and fractional compositions (\%) of IPP/DMAPP ( $C_{5}$ )-, GPP $\left(C_{10}\right)$-, and FPP $\left(C_{15}\right)$-derived isoprenoids from strains NA-MBF2.0 and NAK-MBF2.0. Strains NA-MBF2.0 and NAK-MBF2.0 were transformed with pSNA and pSNAK, respectively, along with pT-MBF2.0. Cultures were incubated at $30^{\circ} \mathrm{C}$ for $48 \mathrm{~h}$ in $2 \mathrm{YT}$ medium containing $2.0 \%$ (v/v) glycerol and were initially induced with $0.5 \mathrm{mM}$ IPTG. The error bars represent the range from three independent experiments 
Table 1 Production of isoprenoid alcohols from recombinant E. coli using NudB, PgpB, and YbjG

\begin{tabular}{llllll}
\hline Phosphatase & $\begin{array}{l}\text { Isopentenol }\left(\mathbf{C}_{\mathbf{5}},\right. \\
\mathbf{m g} / \mathbf{L})\end{array}$ & $\begin{array}{l}\text { Geraniol }\left(\mathbf{C}_{\mathbf{1 0}},\right. \\
\mathbf{m g} / \mathbf{L})\end{array}$ & $\begin{array}{l}\text { Farnesol }\left(\mathbf{C}_{\mathbf{1 5}},\right. \\
\mathbf{m g} / \mathbf{L})\end{array}$ & $\begin{array}{l}\text { Total production } \\
\mathbf{( m g} / \mathbf{L})\end{array}$ & References \\
\hline NudB & 55 & - & - & 55 & {$[13]$} \\
NudB & 2230 & - & - & 2230 & {$[27]$} \\
NudB & 475 & - & - & 1130 & {$[42]$} \\
NudB & 1130 & - & - & 135.5 & {$[43]$} \\
Not specified & - & - & 135.5 & 526.1 & {$[37]$} \\
PgpB/YbjG & - & - & 526.1 & 1430 & {$[49]$} \\
NudB & 1319 & 99 & 12 & 2027 & This study (NA-MBF2.0 strain) \\
NudB & 1105 & - & 922 & 1831 & This study (NA-MBF1.2 strain) \\
NudB & 412 & - & 1419 & & This study (NA-MBF1.2a strain) \\
\hline
\end{tabular}

The "-" represents "not detectable"

dephosphorylates GPP to geraniol. The new promiscuous activity of NudB toward GPP was verified by in vitro study (Additional file 2: Fig. S6).

\section{Identification of enzymes leading to the conversion of alcohols to their derivatives}

Isoprenyl acetate and farnesyl acetate are esters of isoprenol and farnesol, respectively. The formation of esters has not been reported in the production of isopentenol and farnesol [13, 37, 41, 46-49]. Chloramphenicol acetyltransferase (CAT) has been reported to possess nonspecific esterification activity toward some isoprenoid alcohols such as perillyl alcohol [50], geraniol [26], and retinol [51]. Thus, the formation of isoprenyl acetate and farnesyl acetate in strain NA-MBF1.0 was suspected due to the chloramphenicol antibiotic marker $\left(\mathrm{Cm}^{\mathrm{R}}\right)$ gene (cat) in plasmid pSNA. To validate this theory, plasmid pSNAK with a kanamycin antibiotic marker $\left(\mathrm{Km}^{\mathrm{R}}\right)$ gene was used instead of pSNA. The strain NAK-MBF1.0 harboring pT-MBF1.0 and pSNAK produced isoprenoid alcohols (isoprenol, prenol, and farnesol), farnesal, and farnesyl acetate, but no formation of isoprenyl acetate was observed (Fig. 3a). However, the production of farnesyl acetate was still observed in strain NAK-MBF1.0 (Fig. 3a). This indicates that CAT contributes to the esterification of isoprenol to its acetyl ester, but is not responsible for the formation of farnesyl acetate. Hence, some unknown endogenous acetyltransferase in E. coli seems to be involved in the esterification of farnesol into farnesyl acetate (Fig. 1a). The cell growth of NAK-MBF1.0 was slightly lower than that of NA-MBF1.0 (Additional file 1: Fig. S2).

Farnesal is an oxidized product of farnesol. The oxidation of farnesol to farnesal and even to farnesoic acid by alcohol dehydrogenase has been reported in Aedes aegypti
[52]. Oxidation of geraniol to geranial and neral by alcohol dehydrogenases has been reported in E. coli [26]. The formation of farnesal in strains NA-MBF1.0 and NAKMBF1.0 was thus suspected to be a result of oxidation of farnesol by some endogenous alcohol dehydrogenases/ aldehyde reductases. To address this, YjgB, YahK, YddN, and AdhE from E. coli were overexpressed using the vector pTrc99A. YjgB and YahK are NADPH-dependent aldehyde reductases, exhibiting broad substrate specificity toward aldehydes and alcohols [53]. YddN is a NADH-dependent acetaldehyde reductase with a catalytic activity toward medium-chain alcohols [54]. AdhE is an aldehyde-alcohol dehydrogenase, catalyzing the conversion of primary alcohols to aldehydes [55]. Authentic standard farnesol was fed to the culture of $E$. coli strains DH5 $\alpha-\operatorname{Trc}, \mathrm{DH} 5 \alpha-\mathrm{YjgB}$, DH $5 \alpha$-YahK, DH $5 \alpha$-YddN, and DH5 $\alpha$-AdhE harboring plasmids pTrc99A, pT-YjgB, pT-YahK, pT-YddN, and pTAdhE, respectively (Additional file 1: Table S2). The strain DH5 $\alpha$-YjgB significantly transformed farnesol to farnesal (Additional file 1: Table S2). To confirm the dehydrogenase activity of YjgB toward farnesol, farnesol was fed to a culture of mutant strain MG $\triangle Y j g B$ with a deletion of $y j g B$; no farnesal peak was observed in GC analysis of the culture (data not shown).

Similarly, formation of isoprenyl, prenyl, and geranyl acetates in strain NA-MBF2.0 was also suspected due to esterification of isoprenol, prenol, and geraniol, respectively, by the CAT of plasmid pSNA. There was no formation of the isoprenoid acetate esters in the strain NAK-MBF2.0 harboring pT-MBF2.0 and pSNAK (Fig. 3b). The citral produced from strains NA-MBF2.0 and NAK-MBF2.0 is the dehydrogenated product of geraniol formed by YjgB [26]. Cell growth of strain NAK-MBF2.0 was slightly reduced in comparison to that of strain NA-MBF2.0 (Additional file 1: Fig. S2). 
Effects of isp $A$ and nudB operon gene order along with overexpression of $a p h A$ on total titer of the isoprenoid mixture

Since we successfully constructed strains for synthesis of $\mathrm{C}_{5}-\mathrm{C}_{10}$ and $\mathrm{C}_{5}-\mathrm{C}_{15}$ isoprenoid-based biofuel mixtures, we next sought to improve the production of the $\mathrm{C}_{5}-\mathrm{C}_{15}$ isoprenoid-based biofuels mixture. A balanced expression of production pathway genes is critical to achieve high titers of desirable products. There is a fundamental relationship between expression level of genes and their order in an operon. In general, expression of a gene increases as its position in an operon moves closer to a promoter [56]. Positional modulation of the constituent genes in a synthetic operon has significantly enhanced productions of zeaxanthin, taxadiene, and protoilludene in $E$. coli [57-59]. The order of the isp $A$ and $n u d B$ genes in pT-MBF1.0 was reversed to construct pT-MBF1.1 (Fig. 1b). Cell growth of strain NA-MBF1.1 harboring pT-MBF1.1 and pSNA was significantly enhanced in comparison with the growth of strain NA-MBF1.0 (Additional file 1: Fig. S3). The total titer of the isoprenoid mixture from strain NA-MBF1.1 was $1652 \mathrm{mg} / \mathrm{L}$, a twofold increase over the production of strain NA-MBF1.0 $(847 \mathrm{mg} / \mathrm{L})$ (Fig. 4). The significant enhancement of the total production was mainly due to the increased production of $\mathrm{C}_{5}$ products (prenol, isoprenol, and isoprenyl acetate) from 221 to $979 \mathrm{mg} / \mathrm{L}$, whereas the production of $\mathrm{C}_{15}$ products (farnesol, farnesal, and farnesyl acetate) was not significantly different between the strains (Fig. 4). These results show that insufficient expression of NudB from pT-MBF1.0 caused the lower production of $\mathrm{C}_{5}$ products in strain NA-MBF1.0 because of the limited conversion of IPP and DMAPP to $\mathrm{C}_{5}$ products, which would also cause an intracellular accumulation of IPP and DMAPP. The lower cell growth of strain NA-MBF1.0 was presumed to be due to the accumulation of IPP and DMAPP, which are toxic and can inhibit normal cell growth [60].

NudB was thought to convert IPP and DMAPP directly to isoprenol and prenol, respectively [13]. However, a recent study has shown that NudB hydrolyzes IPP and DMAPP into their monophosphate forms, IP and DMAP, respectively, in $E$. coli, and the subsequent hydrolysis of IP and DMAP to isopentenol is performed by other phosphatases such as AphA, Agp, and YqaB [42]. The aphA gene was thus cloned into plasmid pT-MBF1.1 after ispA, resulting in pT-MBF1.2 (Fig. 1b). The strain NA-MBF1.2 harboring pT-MBF1.2 and pSNA produced $2027 \mathrm{mg} / \mathrm{L}$ of total isoprenoid mixture $\left(1105 \mathrm{mg} / \mathrm{L}\right.$ of $\mathrm{C}_{5}$ products and $922 \mathrm{mg} / \mathrm{L}$ of $\mathrm{C}_{15}$ products), which was an increase of 1.2-fold over the production of strain NA-MBF1.1 (Fig. 4). The overexpression of AphA enhanced the production of not only the $C_{5}$ products but also the $C_{15}$

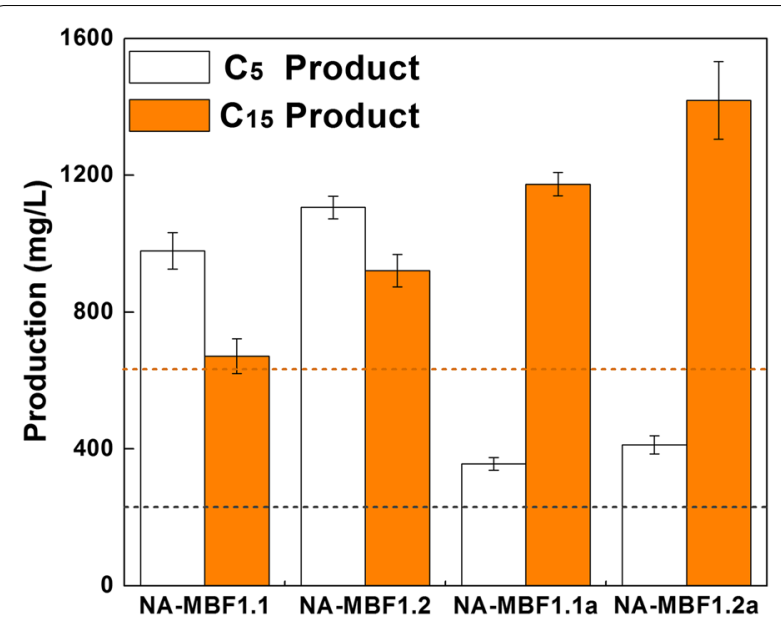

Fig. 4 An optimized synthetic pathway for the production of $C_{5}$ and $\mathrm{C}_{15}$ isoprenoid alcohols and their derivatives. Production of IPP/DMAPP $\left(C_{5}\right)$ - and FPP $\left(C_{15}\right)$-derived isoprenoids from strains NA-MBF1.1, NA-MBF1.2, NA-MBF1.1a, and NA-MBF1.2a harboring pT-MBF1.1, pT-MBF1.2, pT-MBF1.1a, and PT-MBF1.2a, respectively, in addition to PSNA. Cultures were incubated at $30^{\circ} \mathrm{C}$ for $48 \mathrm{~h}$ in $2 \mathrm{YT}$ medium containing $2.0 \%(\mathrm{~V} / \mathrm{V})$ glycerol and were initially induced with $0.5 \mathrm{mM} \mathrm{IPTG}$. The error bars represent the range from three independent experiments. The two dotted lines represent the production of $C_{5}$ products (black) and $C_{15}$ products (orange) from strain NA-MBF1.0

products. This suggests that FPP is sequentially dephosphorylated to farnesol by the cooperation of NudB and AphA, similar to the dephosphorylation of IPP/DMAPP to isopentenol. A significant decrease in farnesol production has been reported in a previous study of an AphA mutant strain [37], indicating the involvement of AphA in farnesol production. The cell growth of strain NAMBF1.2 was slightly reduced in comparison with that of strain NA-MBF1.1 (Additional file 1: Fig. S3). To date, NudB has been only known to have its activity on IPP/ DMAPP and used for production of $\mathrm{C}_{5}$ isoprenoid alcohols (Table 1). However, NudB has a promiscuous activity on isoprenyl diphosphates (IPP/DMAPP, GPP, and FPP), and is successfully applied here for production of mixed isoprenoid alcohols (Table 1). Although George et al. reported $2230 \mathrm{mg} / \mathrm{L}$ of a single $\mathrm{C}_{5}$ alcohol (isoprenol), their production of mixed $\mathrm{C}_{5}$ alcohols (isoprenol, prenol, and isopentanol) was only $1150 \mathrm{mg} / \mathrm{L}$ [41]. Here, we produced the comparable amount of mixed $\mathrm{C}_{5}$ alcohols $(1105 \mathrm{mg} / \mathrm{L})$ in addition to $\mathrm{C}_{15}$ alcohol $(922 \mathrm{mg} / \mathrm{L})$, a total mixed isoprenoid alcohol of $2027 \mathrm{mg} / \mathrm{L}$.

\section{Augmented expression of the idi gene changes the compositional profile of the isoprenoid mixture} The prenol fractions of the isoprenoid mixtures obtained from strains NA-MBF1.1 and NA-MBF1.2 were very 
small compared to those of isoprenol. This is probably due to a lower intracellular level of DMAPP than IPP or a higher specificity of NudB toward IPP than DMAPP. IPP isomerase (IDI) catalyzes the reversible isomerization of IPP to DMAPP and adjusts its ratio to 3:7 [61]. In order to increase the conversion of IPP to DMAPP, the idi gene was cloned downstream of the ispA gene of pT-MBF1.1 and pT-MBF1.2, resulting in pT-MBF1.1a and pT-MBF1.2a, respectively (Fig. 1b). The effect of the additional overexpression of IDI on the compositional profile of the isoprenoid mixture was investigated in strains NA-MBF1.1a and NA-MBF1.2a harboring pTMBF1.1a/pSNA and pT-MBF1.2a/pSNA, respectively (Fig. 4; Additional file 1: Fig. S4). The additional overexpression of IDI drastically changed the compositional profile with a significant decrease and increase in $\mathrm{C}_{5}$ and $\mathrm{C}_{15}$ products, respectively, but a small increase of prenol. IPP-derived products (isoprenol and isoprenyl acetate) of the strains NA-MBF1.1a and NA-MBF1.2a were 303 and $345 \mathrm{mg} / \mathrm{L}$, respectively, which were distinctively lower than 965 and $1086 \mathrm{mg} / \mathrm{L}$ of the corresponding strains NA-MBF1.1 and NA-MBF1.2, (Fig. 4, Additional file 1: Fig. S4). The concentrations of DMAPP-derived prenol in strains NA-MBF1.1a and NA-MBF1.2a were 53 and $67 \mathrm{mg} / \mathrm{L}$, respectively, which were increased more than threefold compared to those in strains NA-MBF1.1 and NA-MBF1.2; however, the absolute increases were only
39 and $47 \mathrm{mg} / \mathrm{L}$, respectively (Additional file 1: Fig. S4). However, the concentrations of FPP-derived products (farnesol, farnesal, and farnesyl acetate) of the strains NA-MBF1.1a and NA-MBF1.2a significantly increased to 1174 and $1419 \mathrm{mg} / \mathrm{L}$, respectively, in comparison with 706 and $922 \mathrm{mg} / \mathrm{L}$ in strains NA-MBF1.1 and NAMBF1.2, respectively (Fig. 4; Table 1; Additional file 1: Fig. S4). The increase in FPP-derived products could be ascribed to an increase in the intracellular levels of DMAPP through the expression of IDI. The FPP synthase IspA possesses a higher $\mathrm{Km}$ with DMAPP $(50 \mu \mathrm{M})$ than with IPP $(4 \mu \mathrm{M})$ [62], and therefore a higher intracellular DMAPP level through the additional overexpression of IDI could be a benefit to divert the flux of isoprenyl diphosphates to FPP. Moreover, the DMAPP would initiate additional FPP synthesis as a reaction-priming molecule (Fig. 5). Although the additional overexpression of IDI increased the prenol fraction in the isoprenoid mixture, there was still a significant difference between the titers of isopentenol (IPP-derived product) and prenol (DMAPP-derived product) (Additional file 1: Fig. S4), which could indicate a preference of NudB for IPP over DMAPP. The cell growths of strains NA-MBF1.1a and NA-MBF1.2a were slightly lower than those of strains NA-MBF1.1 and NA-MBF1.2 (Additional file 1: Fig. S3). The specific total production of the isoprenoid mixtures from strains NA-MBF1.1a and NA-MBF1.2a was
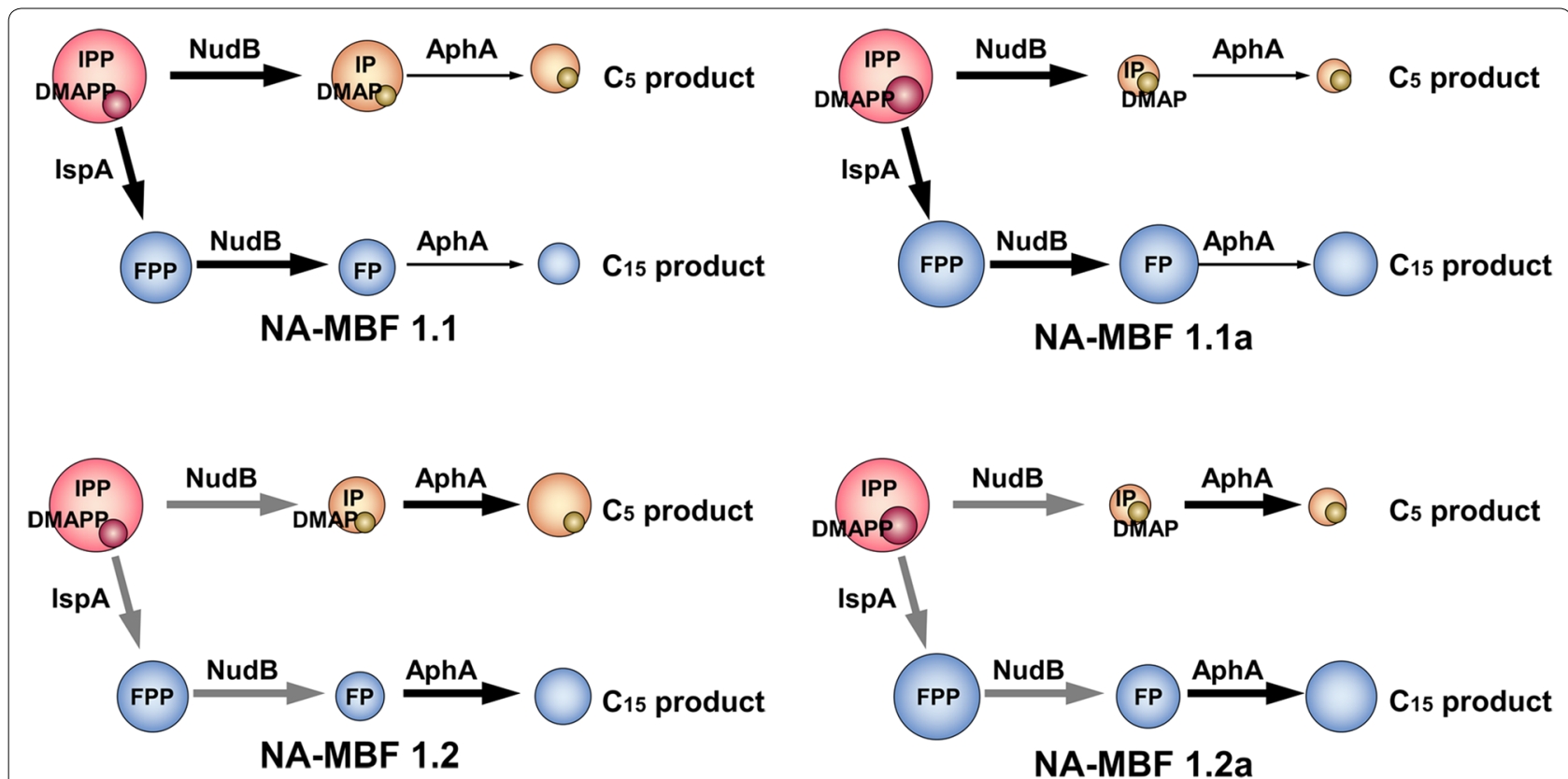

Fig. 5 Scheme of isoprenyl diphosphates flux distribution in engineered E. coli strains with and without additional overexpression of IDI. In strains NA-MBF1.1 and NA-MBF1.2 with no additional overexpression of IDI (left panel), metabolic flux of IPP is mainly directed toward formation of isoprenol through NudB. In strains NA-MBF1.1a and NA-MBF1.2a with additional overexpression of IDI (right panel), the IPP flux is diverted toward synthesis of FPP by IspA, resulting in production of farnesol as the dominant component. The circle area represents the accumulation levels of the intermediates, and the arrows represent the flux directions and the enzyme expression levels 
enhanced over the corresponding strains NA-MBF1.1 and NA-MBF1.2 (Fig. 6). FPP-derived products were a major fraction of the isoprenoid mixtures obtained from strains NA-MBF1.1a and NA-MBF1.2a. This suggests that NudB is an effective phosphatase even with FPP, and the compositional profile of the isoprenoid mixture can be modulated by changes in metabolic flux toward various isoprenyl diphosphates (Fig. 5). It presents an important issue in field of metabolic engineering. Product profile of an enzyme is often restricted by its surroundings such as an intracellular level of metabolites (intermediates or precursors to the enzymatic reaction), and thus a specificity of enzymes is designated in a given specific condition. This study successfully shows a modulation of product profile by implementations of the promiscuous activity of NudB and a change of intracellular synthesis of the precursor isoprenyl diphosphates (IPP/ DMAPP, GPP, and FPP). The maximum titer of farnesol production reported until now is $526 \mathrm{mg} / \mathrm{L}$ [49], while we produced $1419 \mathrm{mg} / \mathrm{L}$ of farnesol in addition to $412 \mathrm{mg} / \mathrm{L}$ of isopentenol. This is the highest titer of farnesol production reported.

\section{Conclusions}

New promiscuous activity of NudB for GPP and FPP was identified for the first time and used for the biosynthesis of isoprenoid mixture biofuels from a cheap carbon source, glycerol, in engineered E. coli strains. A mixture of $C_{5}$ and $C_{15}$ isoprenoid alcohols and their derivatives was achieved in recombinant $E$. coli overexpressing NudB and IspA along with an exogenous MVA pathway. The co-overexpression of the acid phosphatase AphA

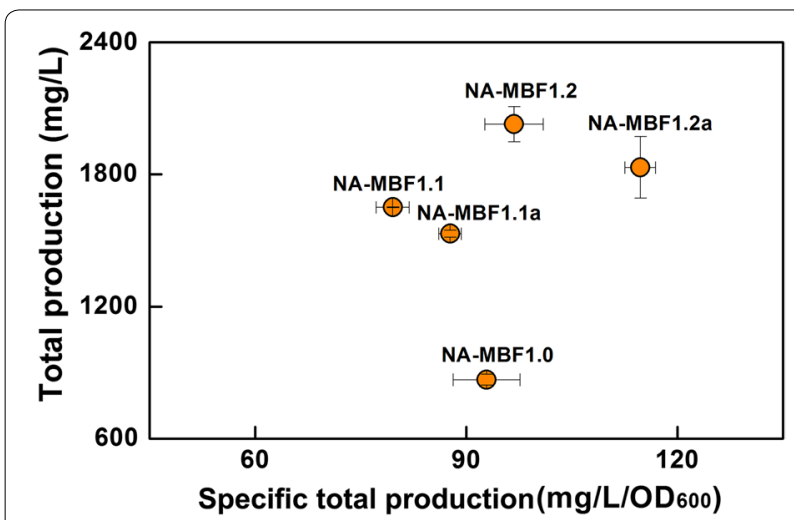

Fig. 6 Total production versus specific total production of isoprenoid mixtures from engineered E. coli strains. Strains NA-MBF1.1, NA-MBF1.2, NA-MBF1.1a, and NA-MBF1.2a are E. coli DH5a strains harboring pT-MBF1.1, pT-MBF1.2, pT-MBF1.1a, and pT-MBF1.2a, respectively, in addition to pSNA. Cultures were incubated at $30^{\circ} \mathrm{C}$ for $48 \mathrm{~h}$ in $2 \mathrm{YT}$ medium containing $2.0 \%(\mathrm{~V} / \mathrm{V})$ glycerol and initially induced with $0.5 \mathrm{mM} \mathrm{IPTG}$. The error bars represent the range from three independent experiments successfully improved production of the $\mathrm{C}_{5}-\mathrm{C}_{15}$ isoprenoid mixture to a maximum titer of $2027 \mathrm{mg} / \mathrm{L}$. The compositional profile of the $\mathrm{C}_{5}-\mathrm{C}_{15}$ isoprenoid mixture can be tailored by overexpression of IDI, resulting in an increase in long carbon chain $\mathrm{C}_{15}$ products with higher energy content than the short carbon chain $\mathrm{C}_{5}$ products. When IspA was replaced with its mutant IspA* that generates GPP, $\mathrm{C}_{10}$ family products were observed in the isoprenoid mixture. All of the isoprenoid mixtures can be good candidates for advanced biofuels and commodity chemicals. This is the first successful report on the production of isoprenoid alcohols and their derivatives in a mixture. This study can serve as the basis for production of other isoprenoid-based mixtures.

\section{Methods}

\section{Strains, media, and culture conditions}

The E. coli DH5 $\alpha$ strain was used as a host for cloning and production of desired compounds. For plasmids construction, cells were grown overnight at $37{ }^{\circ} \mathrm{C}$ in LB (Luria-Bertani) medium (10 g tryptone, 5 g yeast extract, and $10 \mathrm{~g}$ sodium chloride per liter), while cultures for seed preparation and isoprenoid production were grown in $2 \mathrm{YT}$ medium (16 g tryptone, $10 \mathrm{~g}$ yeast extract, and $5 \mathrm{~g}$ sodium chloride per liter). Antibiotics were added to the culture medium at a final concentration of $100 \mathrm{mg} / \mathrm{L}$ of ampicillin and $50 \mathrm{mg} / \mathrm{L}$ of kanamycin or chlorampheni$\mathrm{col}$ as required. To make the seed culture, colonies were inoculated in $5 \mathrm{~mL}$ of $2 \mathrm{YT}$ medium with appropriate antibiotics and grown overnight at $30{ }^{\circ} \mathrm{C}$ with shaking at $250 \mathrm{rpm}$. The production culture was made by inoculating the overnight seed culture into $4 \mathrm{~mL}$ of $2 \mathrm{YT}$ medium to an optical density at $600 \mathrm{~nm}\left(\mathrm{OD}_{600 \mathrm{~nm}}\right)$ of 0.1 of with $2 \%(\mathrm{v} / \mathrm{v})$ of glycerol as the main carbon source. The culture was carried out in two-phases by overlaying $1 \mathrm{~mL}$ of oleyl alcohol over $4 \mathrm{~mL}$ of the culture in order to prevent volatile loss of the products $[41,63]$. The two-phase production culture was initially induced with $0.5 \mathrm{mM}$ isopropyl $\beta$-D-1-thiogalactopyranoside (IPTG) and was incubated for $48 \mathrm{~h}$ with $250 \mathrm{rpm}$ shaking in a rotary shaker at $30{ }^{\circ} \mathrm{C}$. Cellular growth of the culture was measured in terms of $\mathrm{OD}_{600 \mathrm{~nm}}$ using a spectrophotometer. Glass tubes $(2.5 \mathrm{~cm} \times 15 \mathrm{~cm})$ with plastic caps were used for aerobic culture for all experiments conducted in this study. Genomic DNA of E. coli MG1655 was used for PCR amplification of the genes cloned in this study. All strains used in this study are listed in Additional file 1: Table S1.

\section{Plasmid construction}

PCR primers and plasmids used in this study are listed in Additional file 1: Table S1. PCR was performed using Pfu DNA polymerase (SolGent, Daejeon, Korea) 
according to the manufacturer's instructions for amplification of DNA fragments. The ispA [Genbank: NC_000913.3: 440202-441101] fragment was PCR amplified from the genomic DNA of E. coli MG1655 with primers IspA-F and IspA-R, digested with restriction enzymes $S a c \mathrm{I}$ and $\mathrm{Kp} n \mathrm{I}$, and ligated between the corresponding restriction sites of pTrc99A to create pTSBL. For the construction of pT-MBF1.0, $n u d B$ [Genbank: NC_000913.3: 1948180-1948632] was amplified from the genomic DNA with primers NudB-F1 and NudB-R1, digested with BamHI and HindIII, and subsequently inserted between the corresponding restriction sites of pT-SBL. The ispA mutant isp $A^{*}$ was PCR amplified with primers IspA*-F and IspA*-R using the plasmid pT-GPSGES [26] as a template. The PCR product was digested with $\mathrm{KpnI}$ and BamHI and ligated into the corresponding restriction sites of pT-SBL to create pT-MBF2.0. Another fragment of $n u d B$ was PCR amplified from pT-MBF1.0 with primers NudB-F2 and NudB-R2, digested with $N c o \mathrm{I}$ and $S a c \mathrm{I}$, and ligated upstream of ispA in pT-SBL to create pT-MBF1.1. The idi [Genbank: NC_000913.3: 3033065-3033613] fragment was PCR amplified from the genomic DNA with primers Idi-F and Idi-R. The resulting fragment was digested with BamHI and SalI and inserted into the corresponding restriction sites of pT-MBF1.1 to construct pT-MBF1.1a. The DNA fragment of aphA [Genbank: NC_000913.3: 4269414-4270127] was PCR amplified from the genomic DNA with primers of AphA-F and AphA-R, digested with SalI and HindIII, and inserted between the corresponding restriction sites of $\mathrm{pT}$ MBF1.1 and pT-MBF1.1a to create pT-MBF1.2 and pT-MBF1.2a, respectively. The genes overexpressed for transformation of farnesol to farnesal, yjgB [Genbank: NC_000913.3: 4495190-4496209], yahK [Genbank: NC_000913.3: 342884-343933], and $y d d N$ [Genbank: NC_000913.3: 1552828-1553838], were previously cloned in the plasmids pT-YjgB, pT-YahK, and pTYddN, respectively [26]. For construction of pT-AdhE, the DNA fragment of adhE [Genbank: NC_000913.3: 1295446-1298121] was PCR amplified from the genomic DNA with primers adhE-F and adhE-R, digested with EcoRI and BamHI, and inserted between the corresponding restriction sites of pTrc99A. To convert acetyl-CoA to IPP and DMAPP via the mevalonate (MVA) pathway, plasmid pSNA encoding the MVA pathway was previously constructed by Yoon et al. [44]. The plasmid pSNA was constructed by cloning of the top portion of the MVA pathway ( $m v a E$ and $m v a S$ from Enterococcus faecalis) and its bottom portion (mvaK1,
mvaK2, and mvaD from Streptococcus pneumonia, and $i d i$ from E. coli) into the pSTV28 vector under control of a lac promoter. The cat gene encoding chloramphenicol acetyltransferase (CAT) in plasmids pSTV28 and pSNA had been replaced with a kanamycin resistance gene, and the resultant plasmids were named pSTV $28 \mathrm{~K}$ and pSNAK, respectively [26].

\section{Farnesol feeding of the culture}

To investigate the conversion of farnesol to farnesal, $1 \mathrm{~mL}$ of decane containing $1 \mathrm{~g} / \mathrm{L}$ of farnesol was put over $4 \mathrm{~mL}$ of $2 \mathrm{YT}$ medium, with $2 \%(\mathrm{v} / \mathrm{v})$ glycerol as a carbon source, which was inoculated with the recombinant strains DH $5 \alpha-$ Trc, DH $5 \alpha-Y j g B, D H 5 \alpha-Y a h K$, DH $5 \alpha-Y d d N$, or DH5 $\alpha$-AdhE, or the mutant strain MG $\triangle Y j g B$. The culture of each strain was initially induced with $0.2 \mathrm{mM}$ IPTG and incubated at $30^{\circ} \mathrm{C}$ with $250 \mathrm{rpm}$ shaking in a rotary shaker for $48 \mathrm{~h}$. The decane phase was collected at 12-h intervals and subsequently subjected to GC analysis to observe the conversion of farnesol to farnesal.

\section{Analysis of products by GC-MS and GC-FID}

The entire culture broth including oleyl alcohol phase was extracted with ethyl acetate after $48 \mathrm{~h}$ of culture [41]. The ethyl acetate fraction was obtained by highspeed centrifugation and subjected to analysis by GCMS and GC-FID. Formation of isoprenoid mixtures, composed of isoprenol, isoprenyl acetate, prenol, prenyl acetate, geraniol, citral, geranyl acetates, farnesol, farnesal, and farnesyl acetate, was identified by GCMS (GC-MS-QP2010, SHIMADZU, Japan). All products were quantitatively analyzed using GC (Agilent Technologies 7890A) equipped with a flame ionization detector (FID). Samples were injected into a DBWAX123-7032 column (30 m in length, $0.320 \mathrm{~mm}$ in internal diameter, and $0.25 \mu \mathrm{m}$ in film thickness), and nitrogen was used as a carrier gas. The GC oven temperature started at $40{ }^{\circ} \mathrm{C}$ and was raised with a gradient of $5{ }^{\circ} \mathrm{C} / \mathrm{min}$ until $80{ }^{\circ} \mathrm{C}$ and held for $1 \mathrm{~min}$. The temperature was then raised at a rate of $60{ }^{\circ} \mathrm{C} / \mathrm{min}$ until $235{ }^{\circ} \mathrm{C}$ where it was held for $5 \mathrm{~min}$. The detector temperature was maintained at $260^{\circ} \mathrm{C}$. The standard curves for isoprenol, prenol, isoprenyl acetate, geraniol, citral, geranyl acetates, farnesol, farnesal, and farnesyl acetate were constructed for the quantitative calculation of their production (Additional file 1: Fig. S5). 


\section{Additional files}

Additional file 1: Table S1. Primers, plasmids and bacterial strains used in this study. Table S2. Time course analysis of oxidation of farnesol to farnesal in E. coli DH5a-YjgB. Figure S1. GC-FID and GC-MS profile of standard isoprenoid-based alcohols and their derivatives. Figure S2. Comparison of cell growth of the strains NA-MBF2.0, NAK-MBF2.0, NA-MBF1.0, and NAK-MBF1.0. Figure S3. Comparison of cell growth of the strains NA-MBF1.1, NA-MBF1.2, NA-MBF1.1a and NA-MBF1.2a. Figure S4. Percent composition of isoprenoid mixtures obtained from the strains NA-MBF1.1, NA-MBF1.2, NA-MBF1.1a and NA-MBF1.2a. Figure S5. GC-FID standard curves of isoprenoid alcohols and their derivatives.

Additional file 2: Table S3. Primers, plasmids and bacterial strains used in this study. Figure S6. GPP and FPP hydrolyzing assay of NudB.

\section{Abbreviations}

IPP: isopentenyl diphosphate; DMAPP: dimethylallyl diphosphate; GPP: geranyl diphosphate; FPP: farnesyl diphosphate; IP: isopentenyl phosphate; DMAP: dimethylallyl phosphate; GP: geranyl phosphate; FP: farnesyl phosphate; MEP: methylerythritol 4-phosphate; MVA: mevalonate; IPTG: isopropyl $\beta$-D-thiogalactoside; PCR: polymerase chain reaction; GC-FID: gas chromatography-flame ionization detector; GC-MS: gas chromatography-mass spectrometry.

\section{Authors' contributions}

SWK, BZ, and CW conceived the study and designed the experiments. BZ, CW, JBP, SHJ, JEP, and BSH carried out the experiments. BZ, CW, and SWK analyzed the data. BZ, CW, and SWK drafted the manuscript. All authors read and approved the final manuscript.

\section{Author details}

1 Division of Applied Life Science (BK21 Plus Program), PMBBRC, Gyeongsang National University, Jinju 52828, Republic of Korea. ${ }^{2}$ School of Biology and Basic Medical Sciences, Soochow University, Suzhou, People's Republic of China.

\section{Acknowledgements}

Not applicable.

\section{Competing interests}

The authors declare that they have no competing interests.

\section{Availability of data and materials}

Not applicable.

\section{Consent for publication}

Not applicable.

\section{Ethics approval and consent to participate}

Not applicable.

\section{Funding}

This work was supported by the C1 Gas Refinery Program through the NRF funded by the MSI (NRF-2016M3D3A1A01913246) and a Grant (NRF016M1A2A2924237) from the National Research Foundation, Korea. Bakht Zada was supported by scholarships from the BK21 Plus Program, MEST, Korea. Chonglong Wang would like to thank the support of the China Postdoctoral Science Foundation (Grant\#: 2017M610350).

\section{Publisher's Note}

Springer Nature remains neutral with regard to jurisdictional claims in published maps and institutional affiliations.

Received: 1 March 2018 Accepted: 19 July 2018

Published online: 24 July 2018
References

1. Kerr RA. Global warming is changing the world. Science. 2007:316(5822):188-90

2. Peralta-Yahya PP, Keasling JD. Advanced biofuel production in microbes. Biotechnol J. 2010:5(2):147-62.

3. Keasling JD. Manufacturing molecules through metabolic engineering. Science. 2010:330(6009):1355-8.

4. Atsumi S, Liao JC. Metabolic engineering for advanced biofuels production from Escherichia coli. Curr Opin Biotechnol. 2008:19(5):414-9.

5. Nielsen J, Larsson C, van Maris A, Pronk J. Metabolic engineering of yeast for production of fuels and chemicals. Curr Opin Biotechnol. 2013;24(3):398-404

6. Dhingra V, Rao KV, Narasu ML. Current status of artemisinin and its derivatives as antimalarial drugs. Life Sci. 1999:66(4):279-300.

7. Carnesecchi S, Bras-Gonçalves R, Bradaia A, Zeisel M, Gossé F, Poupon M-F, Raul F. Geraniol, a component of plant essential oils, modulates DNA synthesis and potentiates 5-fluorouracil efficacy on human colon tumor xenografts. Cancer Lett. 2004:215(1):53-9.

8. Manfredi KP. Terpenes. Flavors, fragrances, pharmaca, pheromones by Eberhard Breitmaier (University of Bonn). Wiley-VCH, Weinheim. 2006. ix + 214 pp. $6.5 \times 9.5$ in. \$65.00. ISBN 3-527-31786-4. J Nat Prod. 2007;70(4):711-711.

9. Tippmann S, Chen Y, Siewers V, Nielsen J. From flavors and pharmaceuticals to advanced biofuels: production of isoprenoids in Saccharomyces cerevisiae. Biotechnol J. 2013:8(12):1435-44.

10. Peralta-Yahya PP, Zhang F, del Cardayre SB, Keasling JD. Microbial engineering for the production of advanced biofuels. Nature. 2012;488(7411):320-8.

11. Rude MA, Schirmer A. New microbial fuels: a biotech perspective. Curr Opin Microbiol. 2009;12(3):274-81

12. Mack JH, Rapp VH, Broeckelmann M, Lee TS, Dibble RW. Investigation of biofuels from microorganism metabolism for use as anti-knock additives. Fuel. 2014;117:939-43.

13. Chou HH, Keasling JD. Synthetic pathway for production of five-carbon alcohols from isopentenyl diphosphate. Appl Environ Microbiol. 2012;78(22):7849-55

14. Rastogi SC, Heydorn S, Johansen JD, Basketter DA. Fragrance chemicals in domestic and occupational products. Contact Dermatitis. 2001;45(4):221-5

15. Polo MP Crespo R, de Bravo MG. Geraniol and simvastatin show a synergistic effect on a human hepatocarcinoma cell line. Cell Biochem Funct. 2011;29(6):452-8.

16. Akiyama H, Oono T, Huh WK, Yamasaki O, Ogawa S, Katsuyama M, Ichikawa H, Iwatsuki K. Actions of farnesol and xylitol against Staphylococcus aureus. Chemotherapy. 2002;48(3):122-8

17. Chaudhary SC, Alam MS, Siddiqui MS, Athar M. Chemopreventive effect of farnesol on DMBA/TPA-induced skin tumorigenesis: involvement of inflammation, Ras-ERK pathway and apoptosis. Life Sci. 2009:85(5):196-205.

18. Grace MH. Chemical composition and biological activity of the volatiles of Anthemis melampodina and Pluchea dioscoridis. Phytother Res. 2002;16(2):183-5.

19. Goldberg L, Haklai R, Bauer $V$, Heiss A, Kloog Y. New derivatives of farnesylthiosalicylic acid (Salirasib) for cancer treatment: farnesylthiosalicylamide inhibits tumor growth in nude mice models. J Med Chem. 2009:52(1):197-205.

20. Togashi N, Inoue Y, Hamashima H, Takano A. Effects of two terpene alcohols on the antibacterial activity and the mode of action of farnesol against Staphylococcus aureus. Molecules. 2008;13(12):3069.

21. Unlu M, Ergene E, Unlu GV, Zeytinoglu HS, Vural N. Composition, antimicrobial activity and in vitro cytotoxicity of essential oil from Cinnamomum zeylanicum Blume (Lauraceae). Food Chem Toxicol. 2010:48(11):3274-80.

22. Barnard DR, Xue R-D. Laboratory evaluation of mosquito repellents against Aedes albopictus, Culex nigripalpus, and Ochlerotatus triseriatus (Diptera: Culicidae). J Med Entomol. 2004;41(4):726-30.

23. Nickerson KW, Atkin AL, Hornby JM. Quorum sensing in dimorphic fungi: farnesol and beyond. Appl Environ Microbiol. 2006;72(6):3805-13.

24. Rohdich F, Hecht S, Gärtner K, Adam P, Krieger C, Amslinger S, Arigoni D, Bacher A, Eisenreich W. Studies on the nonmevalonate terpene biosynthetic pathway: metabolic role of IspH (LytB) protein. Proc Natl Acad Sci USA. 2002:99(3):1158-63. 
25. Bloch K, Chaykin S, Phillips AH, de Waard A. Mevalonic acid pyrophosphate and isopentenylpyrophosphate. J Biol Chem. 1959;234(10):2595-604

26. Zhou J, Wang C, Yoon S-H, Jang H-J, Choi E-S, Kim S-W. Engineering Escherichia coli for selective geraniol production with minimized endogenous dehydrogenation. J Biotechnol. 2014;169:42-50.

27. Liu W, Xu X, Zhang R, Cheng T, Cao Y, Li X, Guo J, Liu H, Xian M. Engineering Escherichia coli for high-yield geraniol production with biotransformation of geranyl acetate to geraniol under fed-batch culture. Biotechnol Biofuels. 2016;9(1):58.

28. Zhou J, Wang C, Yang L, Choi E-S, Kim S-W. Geranyl diphosphate synthase: an important regulation point in balancing a recombinant monoterpene pathway in Escherichia coli. Enzyme Microb Technol. 2015;68:50-5.

29. Liu W, Zhang R, Tian N, Xu X, Cao Y, Xian M, Liu H. Utilization of alkaline phosphatase PhoA in the bioproduction of geraniol by metabolically engineered Escherichia coli. Bioengineered. 2015;6(5):288-93.

30. Enayetallah AE, Grant DF. Effects of human soluble epoxide hydrolase polymorphisms on isoprenoid phosphate hydrolysis. Biochem Biophys Res Commun. 2006;341(1):254-60.

31. Cao L, Zhang P, Grant DF. An insect farnesyl phosphatase homologous to the N-terminal domain of soluble epoxide hydrolase. Biochem Biophys Res Commun. 2009;380(1):188-92.

32. Cheng AX, Xiang CY, Li JX, Yang CQ, Hu WL, Wang LJ, Lou YG, Chen XY. The rice (E)-beta-caryophyllene synthase (OSTPS3) accounts for the major inducible volatile sesquiterpenes. Phytochemistry. 2007;68(12):1632-41.

33. Schnee C, Kollner TG, Gershenzon J, Degenhardt J. The maize gene terpene synthase 1 encodes a sesquiterpene synthase catalyzing the formation of (E)-beta-farnesene, (E)-nerolidol, and (E, E)-farnesol after herbivore damage. Plant Physiol. 2002;130(4):2049-60.

34. Song L. A soluble form of phosphatase in Saccharomyces cerevisiae capable of converting farnesyl diphosphate into E,E-farnesol. Appl Biochem Biotechnol. 2006;128(2):149-58

35. Faulkner A, Chen X, Rush J, Horazdovsky B, Waechter CJ, Carman GM, Sternweis PC. The LPP1 and DPP1 gene products account for most of the isoprenoid phosphate phosphatase activities in Saccharomyces cerevisiae. J Biol Chem. 1999;274(21):14831-7.

36. Touze T, Blanot D, Mengin-Lecreulx D. Substrate specificity and membrane topology of Escherichia coli PgpB, an undecaprenyl pyrophosphate phosphatase. J Biol Chem. 2008;283(24):16573-83.

37. Chonglong W, Sang-Hwal Y, Ali SA, Young-Ryun C, Jae-Yean K, Eui-Sung C, Seon-Won K. Farnesol production from Escherichia coli by harnessing the exogenous mevalonate pathway. Biotechnol Bioeng. 2010;107(3):421-9.

38. McLennan AG. The Nudix hydrolase superfamily. Cell Mol Life Sci. 2006;63(2):123-43.

39. Mildvan AS, Xia Z, Azurmendi HF, Saraswat V, Legler PM, Massiah MA, Gabelli SB, Bianchet MA, Kang LW, Amzel LM. Structures and mechanisms of Nudix hydrolases. Arch Biochem Biophys. 2005:433(1):129-43.

40. Withers ST, Gottlieb SS, Lieu B, Newman JD, Keasling JD. Identification of isopentenol biosynthetic genes from Bacillus subtilis by a screening method based on isoprenoid precursor toxicity. Appl Environ Microbiol. 2007:73(19):6277-83.

41. George KW, Thompson MG, Kang A, Baidoo E, Wang G, Chan LJG, Adams PD, Petzold CJ, Keasling JD, Soon Lee T. Metabolic engineering for the high-yield production of isoprenoid-based C5 alcohols in E. coli. Sci Rep. 2015:5:11128.

42. Kang A, George KW, Wang G, Baidoo E, Keasling JD, Lee TS. Isopentenyl diphosphate (IPP)-bypass mevalonate pathways for isopentenol production. Metab Eng. 2016;34:25-35

43. Kang A, Meadows CW, Canu N, Keasling JD, Lee TS. High-throughput enzyme screening platform for the IPP-bypass mevalonate pathway for isopentenol production. Metab Eng. 2017;41:125-34.

44. Yoon SH, Lee SH, Das A, Ryu HK, Jang HJ, Kim JY, Oh DK, Keasling JD, Kim SW. Combinatorial expression of bacterial whole mevalonate pathway for the production of beta-carotene in E. coli. J Biotechnol. 2009;140(3-4):218-26.
45. Reiling KK, Yoshikuni Y, Martin VJ, Newman J, Bohlmann J, Keasling JD. Mono and diterpene production in Escherichia coli. Biotechnol Bioeng. 2004:87(2):200-12.

46. George KW, Chen A, Jain A, Batth TS, Baidoo EE, Wang G, Adams PD, Petzold CJ, Keasling JD, Lee TS. Correlation analysis of targeted proteins and metabolites to assess and engineer microbial isopentenol production. Biotechnol Bioeng. 2014;111(8):1648-58.

47. Zheng Y, Liu Q, Li L, Qin W, Yang J, Zhang H, Jiang X, Cheng T, Liu W, Xu X, et al. Metabolic engineering of Escherichia coli for high-specificity production of isoprenol and prenol as next generation of biofuels. Biotechnol Biofuels. 2013;6:57.

48. Liu H, Wang Y, Tang Q, Kong W, Chung WJ, Lu T. MEP pathway-mediated isopentenol production in metabolically engineered Escherichia coli. Microb Cell Fact. 2014;13:135

49. Chonglong W, Ju-Eon P, Eui-Sung C, Seon-Won K. Farnesol production in Escherichia coli through the construction of a farnesol biosynthesis pathway - application of PgpB and YbjG phosphatases. Biotechnol J. 2016;11(10):1291-7.

50. Alonso-Gutierrez J, Chan R, Batth TS, Adams PD, Keasling JD, Petzold CJ, Lee TS. Metabolic engineering of Escherichia coli for limonene and perillyl alcohol production. Metab Eng. 2013;19:33-41.

51. Jang HJ, Ha BK, Zhou J, Ahn J, Yoon SH, Kim SW. Selective retinol production by modulating the composition of retinoids from metabolically engineered E. coli. Biotechnol Bioeng. 2015;112(8):1604-12.

52. Ahmad-Sohdi NA, Seman-Kamarulzaman AF, Mohamed-Hussein ZA, Hassan M. Purification and characterization of a novel NAD(P)+farnesol dehydrogenase from polygonum minus leaves. PLOS ONE. 2015;10(11):e0143310.

53. Pick A, Ruhmann B, Schmid J, Sieber V. Novel CAD-like enzymes from Escherichia coli K-12 as additional tools in chemical production. Appl Microbiol Biotechnol. 2013;97(13):5815-24.

54. Shafqat J, Höög J-O, Hjelmqvist L, Oppermann UCT, Ibáñez C, Jörnvall $H$. An ethanol-inducible MDR ethanol dehydrogenase/acetaldehyde reductase in Escherichia coli. Eur J Biochem. 1999;263(2):305-11.

55. Kessler D, Herth W, Knappe J. Ultrastructure and pyruvate formate-lyase radical quenching property of the multienzymic AdhE protein of Escherichia coli. J Biol Chem. 1992;267:18073.

56. Lim HN, Lee Y, Hussein R. Fundamental relationship between operon organization and gene expression. Proc Natl Acad Sci. 2011;108(26):10626-31.

57. Yang L, Wang C, Zhou J, Kim SW. Combinatorial engineering of hybrid mevalonate pathways in Escherichia coli for protoilludene production. Microb Cell Fact. 2016;15(1):14.

58. Nishizaki T, Tsuge K, Itaya M, Dlkhfkoi N, Yanagawa H. Metabolic engineering of carotenoid biosynthesis in Escherichia coli by ordered gene assembly in Bacillus subtilis. Appl Environ Microbiol. 2007;73(4):1355-61.

59. Ajikumar PK, Xiao WH, Tyo KE, Wang Y, Simeon F, Leonard E, Mucha O, Phon TH, Pfeifer B, Stephanopoulos G. Isoprenoid pathway optimization for Taxol precursor overproduction in Escherichia coli. Science. 2010;330(6000):70-4

60. Martin VJ, Pitera DJ, Withers ST, Newman JD, Keasling JD. Engineering a mevalonate pathway in Escherichia coli for production of terpenoids. Nat Biotechnol. 2003:21(7):796-802.

61. Rohdich F, Zepeck F, Adam P, Hecht S, Kaiser J, Laupitz R, Gräwert T, Amslinger S, Eisenreich W, Bacher A, et al. The deoxyxylulose phosphate pathway of isoprenoid biosynthesis: studies on the mechanisms of the reactions catalyzed by IspG and IspH protein. Proc Natl Acad Sci USA. 2003;100(4):1586-91.

62. Fujisaki S, Nishino T, Katsuki H. Isoprenoid synthesis in Escherichia coli. Separation and partial purification of four enzymes involved in the synthesis1. J Biochem. 1986;99(5):1327-37.

63. Connor MR, Cann AF, Liao JC. 3-Methyl-1-butanol production in Escherichia coli: random mutagenesis and two-phase fermentation. Appl Microbiol Biotechnol. 2010;86(4):1155-64. 